\title{
Spatial Density Network Voronoi Diagram
}

\author{
Jingna Liu* and Ye Zhao
}

Department of Electrical and Electronics Engineering, Shijiazhuang Tiedao University, Hebei Shijiazhuang 050043, China

Department of Mathematics and Physics, Shijiazhuang Tiedao University, Hebei Shijiazhuang 050043, China

\begin{abstract}
A new concept of spatial density network Voronoi diagram was proposed base of spatial density Voronoi diagram. And a discrete construction algorithm for spatial density network Voronoi diagram was presented. Results Spatial density can be used to indicate factors related to density such as conveyance and the traffic conditions. Some properties of spatial density network Voronoi diagram were also introduced. In accordance with discrete construction method, achieved the construction of spatial density network Voronoi diagram. Spatial density network Voronoi diagram is a developed density Voronoi diagram. It both perfected the theory about Voronoi diagrams, and extended the range of applications of Voronoi diagrams. So the study of spatial density Voronoi diagram is of great importance both in theory and practice.
\end{abstract}

Keywords: discrete, construction, voronoi diagram, voronoi polygon.

\section{INTRODUCTION}

When a set of a finite number of points are given in the Euclidean space, the plane are divide into several regions, and Voronoi diagram is the collection of which. Fig. (1) give us a planar ordinary Voronoi diagram with 13 generator points. In 1908, Russian mathematician Georgy Fedoseevich Voronoi defined and studied the general n-dimensional case Voronoi diagrams and discussed the concept of Voronoi diagram historically and geometrically [2]. Voronoi diagrams have been widely applied to space partition problems in various disciplines, from astronomy to geography to zoology. According to statistics, research of Voronoi diagrams involves more than twenty domains and has a widespread influence in the world [3]. Recent application examples of Voronoi diagrams include Voronoi-based cellular automata [4], 3-D adaptive tomography in geophysical prospecting [5], Nicholson, Cua, and Satriano et al. studied earthquake distribution analysis and early warning in 2000, 2004, and 2006 respectively [6-8]. Meguerdichian et al. applied Voronoi diagrams to exposure analysis of wireless sensor network in 2001 [9]. In 2004, Subbeya et al. constructed reservoir modeling in petroleum exploitation [10]. Greco and Nelson et al. put forward environmental risk assessment and remote sensing mapping accuracy assessment with Voronoi diagrams in 2005 [11, 12]. In 2006, Costa, Kolingerova' and Zalik biological applied Voronoi diagrams to structure characterization and domain boundary reconstruction for point datasets $[13,14]$.

In geophysics, real-time risk analysis for earthquake early warning was discussed where magnitude estimation is performed via the Bayesian approach, and the earthquake localization is based on the Voronoi cells [15]. In geography, some of the early applications of Voronoi diagrams in market area analysis can be dated back to the 18th century [16, 17]. Economic geography and urban geography problems are major application areas of Voronoi diagrams in geography, for example, modeling retail trade areas [18] and solving locational optimization problems [19].

However, all kinds of Voronoi diagrams developed in Euclidean space. In fact, in many practical applications, the space is not ideal as Euclidean space. Spatial density also plays an important role. To take city Voronoi diagram for an example. The time from one point to another, relates either to the distance between the two points or the means of conveyance and the traffic. The traditional Euclidean space could not indicate those important points. In 2012, the conception of density Voronoi Diagram was first presented by Y. Zhao [20].

Spatial density Voronoi diagram perfects the theory about Voronoi diagrams, and the range of applications of Voronoi diagrams is extended. That makes great effort to the Voronoi diagram research and development. In this paper, density Voronoi Diagram is confined to network, and some new properties and conclusions of density Voronoi Diagram are discussed.

\section{PRELIMINARIES}

In this section, we review briefly some notions and results related to Voronoi diagrams.

A planar ordinary distance: We consider a finite number, $n$, of points in the Euclidean plane, and assume that $2<n<+\infty$. The $\mathrm{n}$ points are labeled by $p_{1}, p_{2}, \cdots, p_{n}$ with 
the Cartesian coordinates $\left(x_{11}, x_{12}\right), \ldots,\left(x_{n 1}, x_{n 2}\right)$ or location vectors $x_{1}, x_{2}, \cdots, x_{n}$. The $\mathrm{n}$ points are distinct in the sense that $x_{i} \neq x_{j}$ for $i \neq j, i, j \in I_{n}=\{1,2, \ldots, n\}$. Let $\mathrm{p}$ be an arbitrary point in the Euclidean plane with coordinates $\left(x_{1}, x_{2}\right)$ or a location vector $x$. Then the Euclidean distance between $p$ and $p_{i}$ is given by

$$
d\left(p, p_{i}\right)=\left\|x-x_{i}\right\|=\sqrt{\left(x_{1}-x_{i 1}\right)^{2}+\left(x_{2}-x_{i 2}\right)^{2}}
$$

If $p_{i}$ is the nearest point from $p$ or $p_{i}$ is one of the nearest points from $p$, we have the relation $\left\|\mid x-x_{i}\right\| \leq\left\|x-x_{j}\right\|$ for $i \neq j, i, j \in I_{n}$. In this case, $p$ is assigned to $p_{i}$. Therefore, Definition is written mathematically as follows. In the literature, a generator point is sometimes referred to as a site, as shown in Fig. (1).

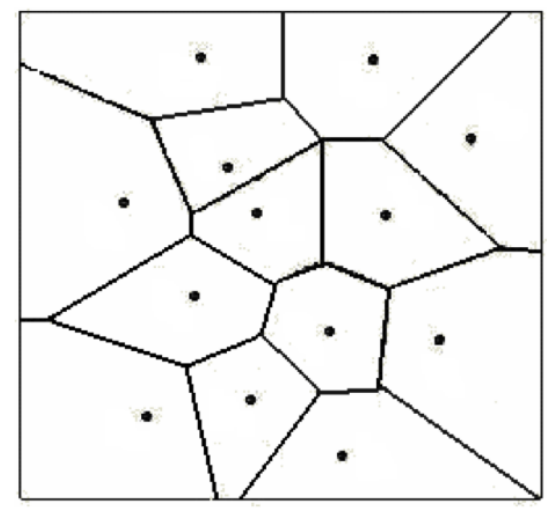

Fig. (1). Planar ordinary voronoi viagram with 13 generator points.

\subsection{A Planar Ordinary Voronoi Diagram}

Let $P=\left\{p_{1}, p_{2}, \cdots, p_{n}\right\} \subset R^{2}$, where $2 \leq n<+\infty$ and $x_{i} \neq x_{j}$ for $i \neq j, i, j \in I_{n}$. We call the region given by

$V\left(p_{i}\right)=\left\{x \mid\left\|x-x_{i}\right\| \leq\left\|x-x_{j}\right\|\right.$ for $\left.j \neq i, j \in I_{n}\right\}$

the planar ordinary Voronoi polygon associated with $p_{i}$ (or the Voronoi polygon of $p_{i}$ ), and the set given by

$\mathrm{V}=\left\{V\left(p_{1}\right), V\left(p_{2}\right), \cdots, V\left(p_{n}\right)\right\}$

the planar ordinary Voronoi diagram generated by $P$ (or the Voronoi diagram of $P$ ). We call $p_{i}$ of $V\left(p_{i}\right)$ the generator point or generator of the ith Voronoi polygon, and the set $P=\left\{p_{1}, p_{2}, \cdots, p_{n}\right\}$ the generator set of the Voronoi diagram $\mathrm{V}$ (in the literature, a generator point is sometimes referred to as a site).

\subsection{An Ordinary Voronoi Diagram $\mathbf{I n} \mathbf{R}^{\mathrm{m}}$}

Let $P=\left\{p_{1}, p_{2}, \cdots, p_{n}\right\} \subset R^{m}, \quad$ where $\quad 2 \leq n<+\infty \quad$ and $x_{i} \neq x_{j}$ for $i \neq j, i, j \in I_{n}$. We call the region

$$
\begin{aligned}
& V\left(p_{i}\right)=\left\{x \mid\left\|x-x_{i}\right\| \leq\left\|x-x_{j}\right\| \text { for } j \neq i, j \in I_{n}\right\} \\
& =\bigcap_{j \in I_{n} \backslash\{i\}} H\left(p_{i}, p_{j}\right)
\end{aligned}
$$

the m-dimensional ordinary Voronoi polyhedron associated with $p_{i}$, and the set $\mathrm{V}=\left\{V\left(p_{1}\right), V\left(p_{2}\right), \cdots, V\left(p_{n}\right)\right\}$ the $m$ dimensional ordinary Voronoi diagram generated by $P$.

\subsection{Network Voronoi Diagram}

A Network Voronoi Diagram is a special kind of Voronoi diagram constructed on spatial networks. The decomposition is based on the connection of the discrete objects rather than Euclidean distance. In the Network Voronoi Diagram, the Voronoi polygon changes to a set of road segments termed Network Voronoi polygon, and the edges of the polygons also shrink to some midpoints, termed border points, of the road network connection between two objects of interest. Besides the objects of interest $(p)$, a Network Voronoi Diagram also includes some road network intersections $(n)$ and border points $(b)$. According to the properties of a Voronoi diagram, from border points to a pair of adjacent objects is equidistant $\left(e . g\right.$. dis $\left.\left(b_{7}, p_{1}\right)=\operatorname{dis}\left(b_{7}, p_{3}\right)\right)$. The blue line segments is the Voronoi region of generator point $p_{1}$, the red line segments is the Voronoi region of generator point $p_{2}$, and the green line segments is the Voronoi region of generator point $p_{3}$.

\subsection{Spatial Density Voronoi Diagram}

Let $P=\left\{p_{1}, p_{2}, \cdots, p_{n}\right\} \subset R^{2}$, where $2 \leq n<+\infty \quad$ and $x_{i} \neq x_{j}$ for $i \neq j, i, j \in I_{n}$. And distribution function $z=f(x$, $y$ ) is integrable in density space. We call the region

$V_{f}\left(p_{i}\right)=\bigcap_{j \neq i}\left\{p \mid d_{f}\left(p, p_{i}\right) \leq d_{f}\left(p, p_{i}\right), j \neq i, j \in I_{n}\right\}$

the spatial density Voronoi polygon associated with $p_{i}$ based on density distribution function $f$ (or the spatial density Voronoi polygon of $p_{i}$ ), and the set given by

$\operatorname{Vor}_{f}=\left\{V_{f}\left(p_{1}\right), V_{f}\left(p_{2}\right), \cdots, V_{f}\left(p_{n}\right)\right\}$

the spatial density Voronoi diagram generated by $P$ based on density distribution function $f$ (or the spatial density Voronoi diagram of $P$ ). We call $p_{i}$ of $V\left(p_{i}\right)$ the generator point or generator of the $i$ th spatial density Voronoi polygon, and the set $P=\left\{p_{1}, p_{2}, \cdots, p_{n}\right\}$ the generator set of the spatial density Voronoi diagram. We call the set given by $V\left(p_{\mathrm{i}}\right) \bigcap_{i \neq j} V_{z}\left(p_{\mathrm{j}}\right)$ spatial density Voronoi edges. And points of intersection of the spatial density Voronoi edges are called spatial density Voronoi vertex. Fig. (2) showed a spatial density Voronoi with 300 generators which generate randomly based on density distribution function $f=3(x+300)+2$ $(y+500)$. 


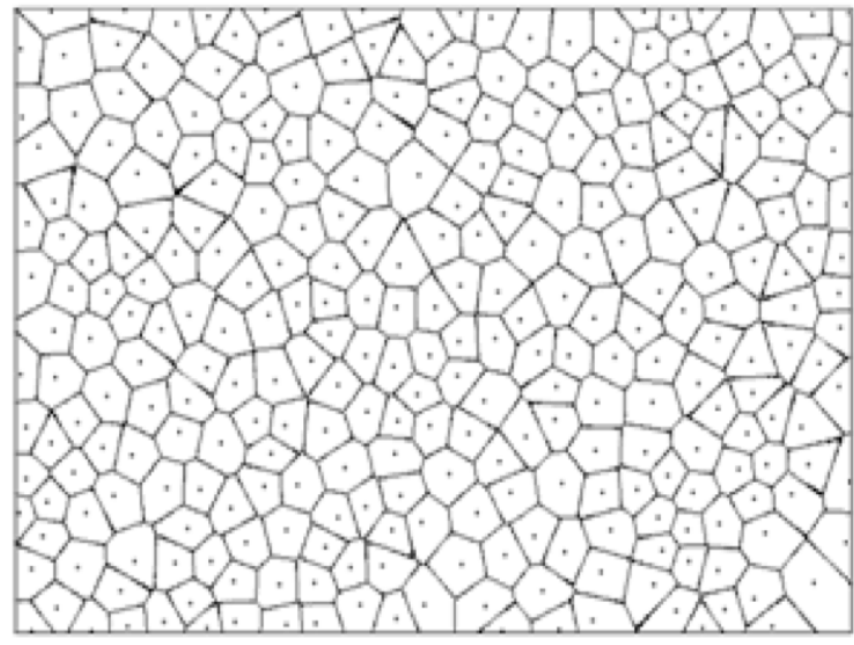

Fig. (2). Spatial density voronoi with 300 generators which generate randomly based on density distribution function $f=3(x+300)+2$ $(y+500)$.

\section{SPATIAL DENSITY NETWORK VORONOI DIA- GRAM}

\subsection{Spatial Density Network Distance}

$z=f(x, y) \geq 0$ is a continuous function on a plane. Consider a planar geometric graph $G(N, L)$ consisting of a set of nodes $N=\left\{p_{1}, p_{2}, \cdots, p_{n}, p_{n+1}, \ldots, p_{l}\right\}$ and a set of links $L=\left\{l_{1}, \cdots, l_{k}\right\}$ which form a connected component. Assume that $G(N, L)$ is a nondirected graph. On $G(N, L)$ we define the distance from a point $p$ on a link in $L$ to a node $p_{i}$ in $\mathrm{N}$ by the spatial density distance from $p$ to $p_{i}$. We call this distance the spatial density network distance, and denote it by $d_{f-n e t}\left(p, p_{i}\right)$. We call a geometric graph $G(N, L)$ with the spatial density network distance a spatial density network, and denote it by $\mathbf{N}_{f}(N, L)$.

\subsection{Spatial Density Network Voronoi Diagram}

It should contain summary of the research work performed, result interpretations and finally their conclusion.

\section{CONCLUSION}

\subsection{Results and Discussion}

Consider that a space $\mathbf{S}$ is given by the node set $\mathrm{N}$, called a node space of a spatial density network $\mathbf{N}_{f}(N, L)$.

Let

$$
\begin{aligned}
& \operatorname{Dom}_{f}\left(p_{i}, p_{j}\right)= \\
& \left\{\begin{array}{l}
p_{k} \mid p_{k} \in N ; \\
d_{f-n e t}\left(p, p_{i}\right) \leq d_{f-n e t}\left(p, p_{i}\right)
\end{array}\right\}, i \neq j \\
& b_{f}\left(p_{i}, p_{j}\right)= \\
& \left\{p_{k} \mid p_{k} \in N ; d_{f-n e t}\left(p, p_{i}\right)=d_{f-n e t}\left(p, p_{i}\right)\right\} .
\end{aligned}
$$

We call $\operatorname{Dom}_{f}\left(p_{i}, p_{j}\right)$ the dominance node set of $p_{i}$ over $p_{j}$ and $b_{f}\left(p_{i}, p_{j}\right)$ the bisector node set between $p_{i}$ and $p_{j}$.

We define

$V_{f-\text { node }}\left(p_{i}\right)=\bigcap_{j \in I_{n} \backslash\{i\}} \operatorname{Dom}_{f}\left(p_{i}, p_{j}\right)$

and let

$V_{f}\left(P, d_{f-n e d}, N\right)=V_{f-n n}=$

$\left\{V_{f-\text { node }}\left(p_{1}\right), \cdots, V_{f-\text { node }}\left(p_{n}\right)\right\}$.

We call the set $V_{f-n n}$ the spatial density network Voronoi node diagram generated by the node set $P$ in the node density space $N_{f}$, and call the set $V_{f-n o d e}\left(p_{i}\right)$ the spatial density network Voronoi node set associated with the node $p_{i}$.

Property 1 In the Network Voronoi Diagram with density function, the Voronoi polygon changes to a set of road segments termed Network Voronoi polygon, and the edges of the polygons also shrink to some midpoints, termed border points, of the road network connection between two objects of interest.

Property 2 Geometrically, spatial density network distance between $p_{i}$ and $p_{j}$ is considered function $f$ is defined integral over $\left[p_{i}, p_{j}\right]$ on network.

Property 3 As a result that continuous functions's definite integral over $[a, b]$ exists, spatial density network distance is exists if functions $f$ is continuous.

Property 4 If functions $f$ is integrable on $[a, b]$, then its mean value on $[a, b]$ exists. So spatial density network Voronoi diagram exists when functions $f$ is integrable.

\section{DISCRETE CONSTRUCTION OF VORONOI DIA- GRAM}

\subsection{Discrete Distance}

For each pixel $p(x, y)$ on the screen, where $0 \leq x<k$, $0 \leq y<l, x, y \in \mathrm{N}$, and $\mathrm{k}$ and 1 are the maximum value of abscissa and ordinate respectively. The distance between two pixels $p_{1}\left(x_{1}, y_{1}\right)$ and $p_{2}\left(x_{2}, y_{2}\right)$ are defined by

$d\left(p_{1}, p_{2}\right)=\sqrt{\left(x_{1}-x_{2}\right)^{2}+\left(y_{1}-y_{2}\right)^{2}}$

\subsection{Discrete Voronoi Diagram}

Let $P=\left\{p_{1}, p_{2}, \cdots, p_{n}\right\}, p_{i}$ is the pixel on the screen, $i \in I_{n}$. We call the region

$$
\begin{aligned}
& V_{n}\left(p_{i}\right)= \\
& \left\{p \mid \begin{array}{cc}
d\left(p, p_{i}\right)<d\left(p, p_{j}\right) & i \neq j \\
d\left(p, p_{i}\right)=d\left(p, p_{j}\right)<d\left(p, p_{k}\right) & i<j, i, j \neq k
\end{array}\right\}
\end{aligned}
$$


the discrete Voronoi polygon associated with $p_{i}$, and the set given by $\mathbf{V}=\left\{V_{n}\left(p_{1}\right), V_{n}\left(p_{2}\right), \cdots, V_{n}\left(p_{n}\right)\right\}$ the discrete Voronoi diagram generated by $P$, and the set $P=\left\{p_{1}, p_{2}, \cdots, p_{n}\right\}$ the generator set of the discrete Voronoi diagram.

Discrete Voronoi Diagram Now we take 3 generator points as the example, and construct Voronoi diagram using discrete algorithm. First, we assign different colors for different generators, as shown in Fig. (3a).

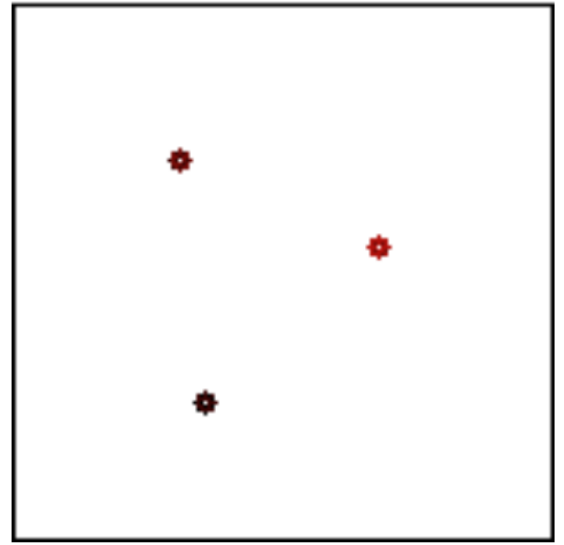

Fig. (3a). The spatial density distance based on density distribution function in space.

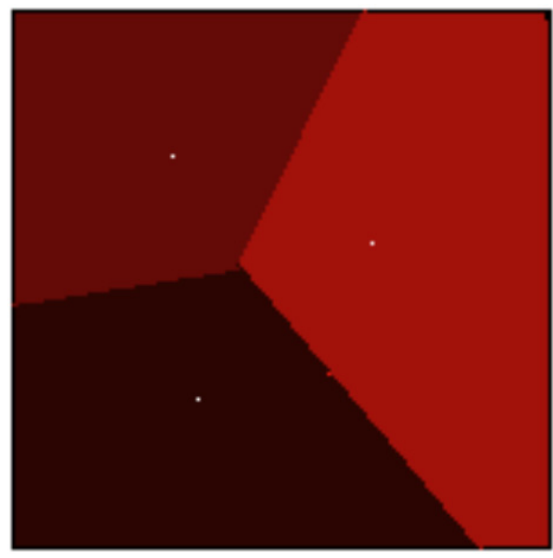

Fig. (3b). Stop painting when all pixels on the screen are painted.

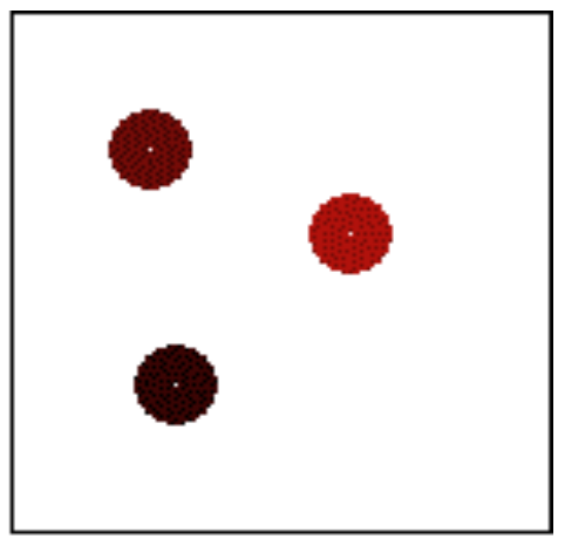

Fig. (3c). Expanding centered on respective generators.

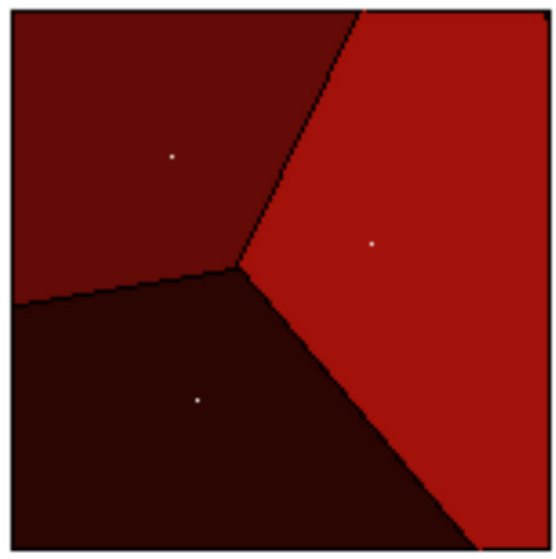

Fig. (3d). Construction of the voronoi edges.

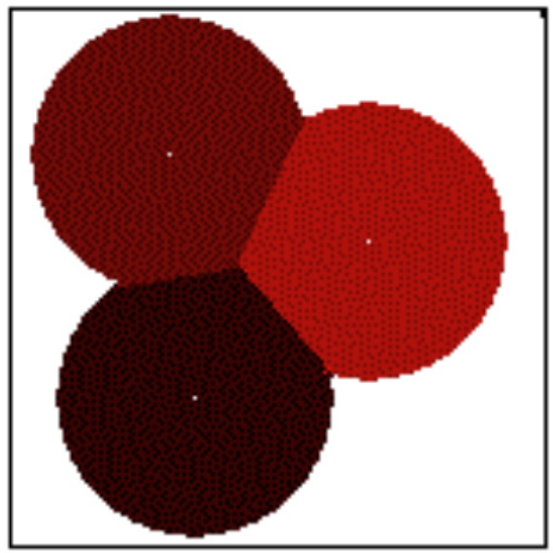

Fig. (3e). Judging before painting.

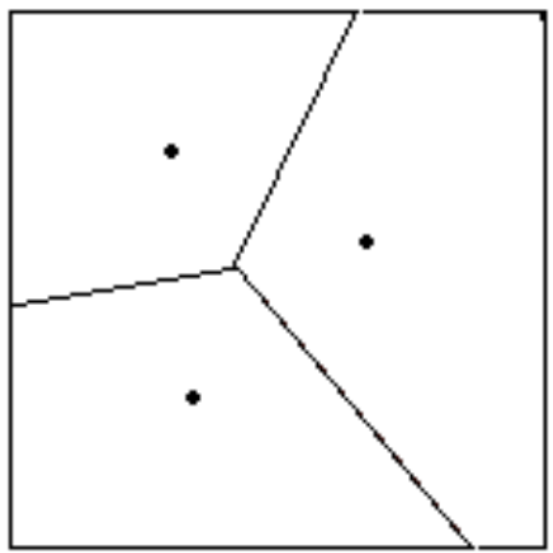

Fig. (3f). Voronoi diagram constructed with discrete algorithm.

Then expand centered on respective generators. In this process, every pixel we consider should be painted with the right color that is concordant with that of its generator, as shown in Fig. (3b). Before paint every pixel, the color of the pixel should be judged. Only those pixels with white color are be painted. And if the pixel which is going to be painted is already a color one, proceed to next step, as shown in Fig. (3c). The procedure end when all points on screen are marked color, as shown in Fig. (3d). When all points on screen are marked color, the regions with different color form the Voronoi edges. To make the region boundaries visible, we do progressive scanning on full screen. Each time 
we record the color value of current pixel, and compare to the previous one. When two adjacent pixels are different color, one of them is marked by black color (such as the first one is turned to black). The black pixels construct the Voronoi edges, as shown in Fig. (3e). Do the second progressive scanning full screen. This time, we retain those black pixels, and turn other pixels to white color. Now we get the Voronoi diagram, as shown in Fig. (3f). Figs. (3a-f) gave a production process of Voronoi diagram with 3 generators, and the main technical steps of construction of Voronoi diagram are described in detail as following:

\subsection{Discrete Network Voronoi Diagram}

Fig. (4a) shows a network with 3 generator points on it in space. Firstly, different colors were assigned for different generator points, and black color for road segments. As shown in Fig. (4b), blue color was assigned for generator point $p_{1}$, red color was assigned for generator point $p_{2}$, and green color was assigned for generator point $p_{3}$. Then take generator points as the centre and draw circles. Unlike the discrete algorithm of planar ordinary Voronoi diagram, we only need to consider four points around generator point: the upper one, the lower one, the left one and the right one, and only need to consider those points on road segments. That is to say, we only assign color for a point with black color. This will greatly improve the efficiency of construction. The procedure end when all points with black color on screen are marked color. And the Network Voronoi diagram was got [22].

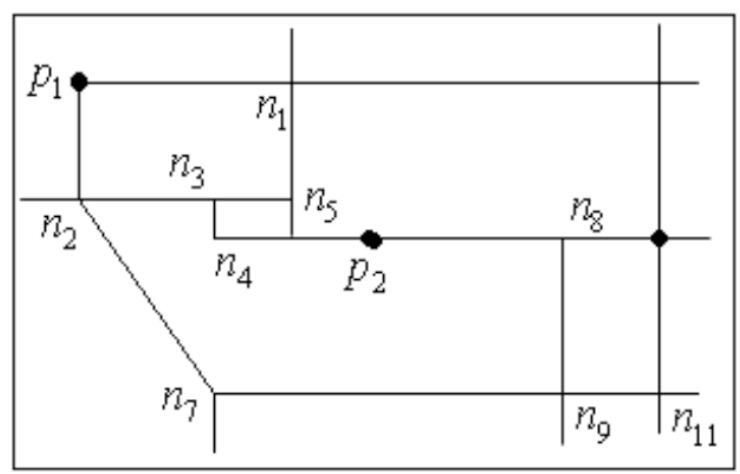

Fig. (4a). 3 Generator points on network in space.

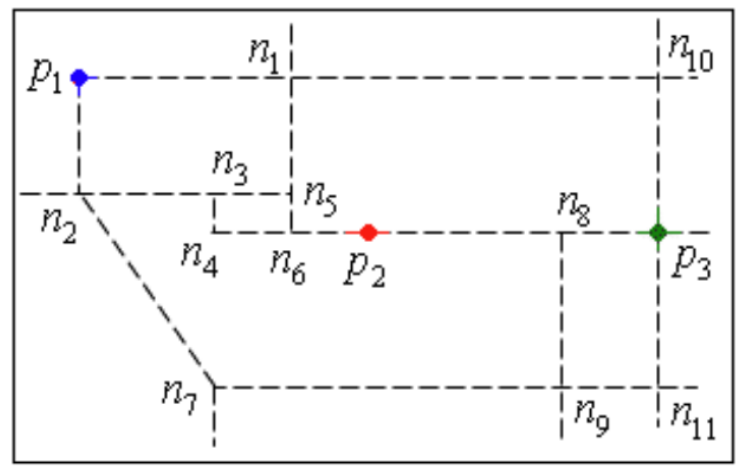

Fig. (4b). Different colors were assigned for different generator points.

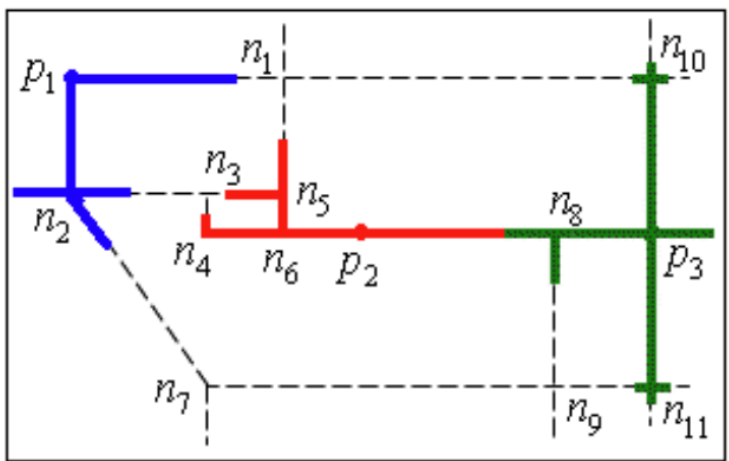

Fig. (4c). Continue drawing circles.

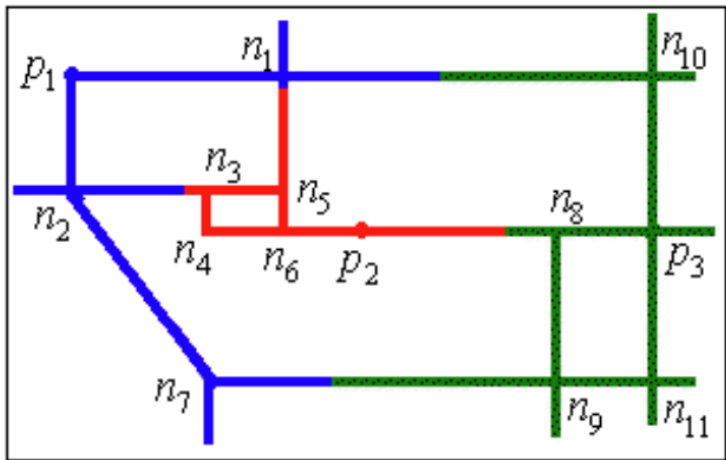

Fig. (4d). Network voronoi diagram.

Give $n$ generator points on the screen, and expand centered on respective generators. In the process of painting, if there still white pixel, painted it with the right color, otherwise delete this generator point from treating list. Figs. (5c and 5d) shows the judge processing, where the point $p$ would be deleted.

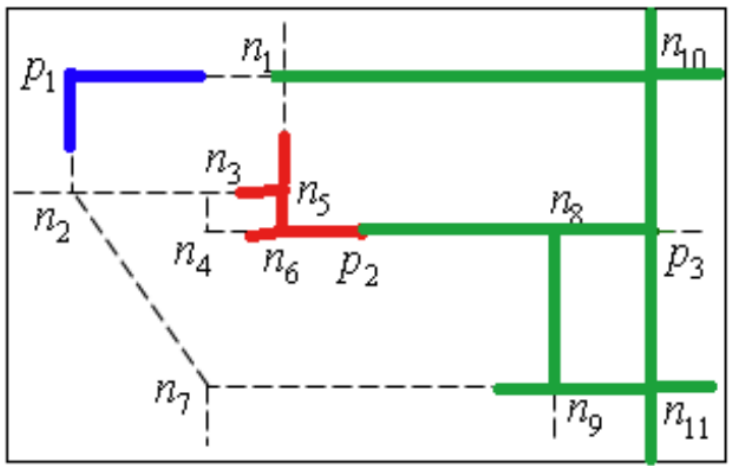

Fig. (5a). Draw circles with spatial density distance as radius.

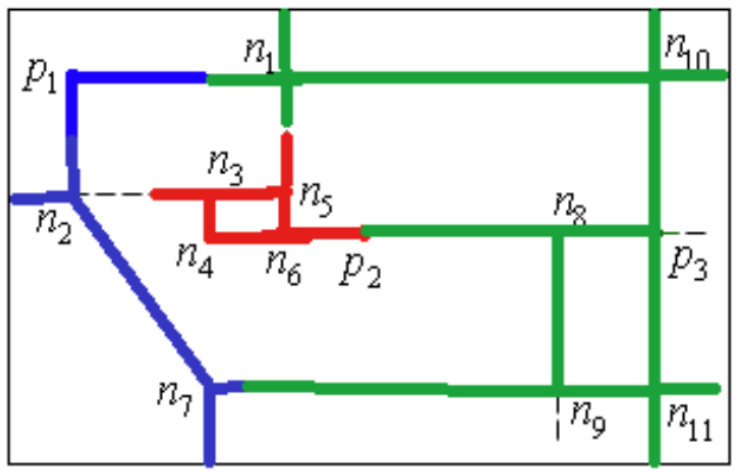

Fig. (5b). Spatial density network voronoi diagram. 


\section{CONSTRUCTION OF DENSITY NETWORK VO- RONOI DIAGRAM}

According to the discrete method, scan the screen. And operation performed on each pixel point in a way that compute the spatial density network distance between the pixel and each generator point denoted by $\mathrm{d}_{f}\left(p_{1}, p_{2}\right)$ on network. Then judge the pixel belongs to which density network Voronoi polygon. The spatial density network Voronoi diagram is got when scanning is over.

Particular way is: First, we assign different colors $c_{i}$ for each generator $p_{i}$. Then scan the screen, deal with those pixel points $q_{i}$, which still not be painted. If it belongs to the density network Voronoi polygon associated with $p_{i}$, mark the pixel with the color of $p_{i}$. When all points on screen are marked color, the regions with different color form the spatial density network Voronoi edges. Then we can get the spatial density network Voronoi diagram.

We still take Fig. (5) for example. There are a network with 3 generator points on it in space shown in Fig. (5a). Firstly, different colors were assigned for different generator points, and black color for road segments as shown in Fig. (5b). Suppose there is function $f(x, y)=x+y$ on the plane. Then take generator points as the centre and draw circles with spatial density distance as radius Fig. (5a). The procedure end when all points with black color on screen are marked color. Finally, the spatial density network Voronoi diagram was got.

\section{CONCLUSION}

A new concept and some properties of spatial density network Voronoi diagram was proposed in this paper. The planar ordinary network Voronoi diagram can be regarded as the generalization of the spatial density network Voronoi diagram with the spatial density function $f=1$. Construction of spatial density network Voronoi diagram is also presented, and examples implementation are given in the end of the paper. Spatial density network Voronoi diagram has better theoretical guiding significance and higher application values.

\section{CONFLICT OF INTEREST}

The authors confirm that this article content has no conflict of interest.

\section{ACKNOWLEDGEMENTS}

This work was supported in part by a grant from the Hebei natural science foundation (A2014210140), the Hebei Educational Committee Foundation (Z2013113), the Hebei Educational Committee Foundation (Z2013061).

\section{REFERENCES}

[1] Boots, B.N., 1980. Weighting Thiessen polygons. Economic.Geography 56, 248-259
[2] Anton, F., Mioc, D., Gold, C.M., 1998. Dynamic additively weighted Voronoi diagrams made easy. In: Proceedings of the Tenth Canadian Conference on Computational Geometry (CCCG'98), Montreal, Canada, p. 2.

[3] Okabe, A., Boots, B., Sugihara, K., Chiu, S.N., 2000. Spatial Tessellations: Concepts and Applications of Voronoi Diagrams, second ed. Wiley, Chichester, UK, 671pp.

[4] Shi, W., Pang, M.Y.C., 2000. Development of Voronoi-based cellular automata - an integrated dynamic model for geographic information systems. International Journal of Geographical Information Science 14, 455-474.

[5] Bo" hm, G., Galuppo, P., Vesnaver, A., 2000. 3D adaptivetomography using Delaunay triangles and Voronoi polygons.Geophysical Prospecting 48, 723-744.

[6] Nicholson, T., Sambridge, M., Gudmundsson, O'., 2000. On entropy and clustering in earthquake hypocenter distributions. Geophysical Journal International 142, 37-51.

[7] Cua, G.B., 2004. Creating the virtual seismologist: developments in ground motion characterization and seismic early warning. Unpublished Ph.D. Dissertation, California Institute of Technology, Pasadena, California, 408pp.

[8] Satriano, C., Lomax, A., Zollo, A., 2006. Optimal, real-time earthquake location for early warning. Geophysical Research Abstracts 8,08883 .

[9] Meguerdichian, S., Koushanfar, F., Qu, G., Potkonjak, M., 2001. Exposure in wireless Ad-Hoc sensor networks. In: Proceedings of the Seventh Annual International Conference on Mobile Computing and Networking, Rome, Italy, pp. 139-150.

[10] Subbeya, S., Christiea, M., Sambridge, M., 2004. Prediction under uncertainty in reservoir modeling. Journal of Petroleum Science and Engineering 44, 143-153.

[11] Greco, F., Lawson, A.B., Cocchi, D., Temples, T., 2005. Some interpolation estimators in environmental risk assessment for spatially misaligned health data. Environmental and Ecological Statistics 12, 379-395.

[12] Nelson, T., Boots, B., Wulder, M.A., 2005. Techniques for accuracy assessment of tree locations extracted from remotely sensed imagery. Journal of Environmental Management 74, 265-271.

[13] Costa, L., Rocha, F., Lima, S., 2006. Characterizing polygonality in biological structures. Physical Review E 73, 1-8.

[14] Kolingerova', I., Zalik, B., 2006. Reconstructing domain boundaries within a given set of points, using Delaunay triangulation. Computers \& Geosciences 32, 1310-1319.

[15] Iervolino, I., Convertito, V., Giorgio, M.,Manfred, G., Zollo, A., 2006. Real-time hazard analysis for earthquake early warning.In Proceedings of the First European Conference on Earthquake Engineering and Seismology, Geneva, Switzerland, Paper no. 850.

[16] Shieh, Y.-N., 1985. K.H. Rau and the economic law of market areas. Journal of Regional Science 25, 191-199.

[17] Okabe, A., Boots, B., Sugihara, K., Chiu, S.N., 2000. Spatial Tessellations: Concepts and Applications of Voronoi Diagrams, second ed. Wiley, Chichester, UK, 671pp.

[18] Boots, B., South, R., 1997. Modeling retail trade areas using higherorder, multiplicatively weighted Voronoi diagrams. Journal of Retailing 73, 519-536.

[19] Pinliang Dong. Computers \& Geosciences. Generating and updating multiplicatively weighted Voronoi diagrams for point, line and polygon features in GIS. 34 (2008) 411-421.

[20] Ye Zhao, Shujuan Liu, Youhui Zhang. Spatial Density Voronoi Diagram and Construction. Journal of Computers. Vol 7, (2012), 2007-2014, Aug 2012.

[21] Yili Tan, Ye Zhao, Yourong Wang. Power Network Voronoi Diagram and Dynamic Construction.Journal of Networks. Vol.7, No 4 (2012),675-682.

[22] Wu Ran,Ye Zhao. Discrete Construction of Network Voronoi Diagram. ICMCI'2010. : 630-632.

\footnotetext{
Received: September 16, 2014

(C) Liu and Zhao; Licensee Bentham Open.
}

Revised: December 23, 2014

Accepted: December 31, 2014

This is an open access article licensed under the terms of the Creative Commons Attribution Non-Commercial License (http://creativecommons.org/licenses/by-nc/3.0/) which permits unrestricted, non-commercial use, distribution and reproduction in any medium, provided the work is properly cited. 